\title{
EFFECT OF COMBINATION OF CEFSULODIN AND MECILLINAM
}

\author{
MASAHIRo Kondo and KanjI TsuchiYa \\ Central Research Division, Takeda Chemical Industries, Ltd., Osaka, Japan
}

(Received for publication February 6, 1981)

\begin{abstract}
The effect of cefsulodin in combination with mecillinam was examined against a wide range of bacterial species. The antibacterial spectrum was widened by the combination of cefsulodin and mecillinam in the ratio of 5:1 and 10:1. In overall observations, in the in vitro test, a synergistic effect against clinical isolates was found on Klebsiella pneumoniae, Citrobacter freundii, Enterobacter cloacae, Serratia marcescens, Proteus mirabilis and Proteus vulgaris, and an additive effect was found on Staphylococcus aureus, Escherichia coli, Proteus morganii, Proteus rettgeri, Proteus inconstans and Pseudomonas aeruginosa. In in vivo tests, a synergistic effect was observed on $S$. marcescens TN 66 and $K$. pneumoniae DT infections and an additive effect was observed on S. aureus 308 A-1, E. coli O-111 and T-7, C. freundii TN 518, E. cloacae TN 603, $P$. vulgaris GN 4712, $P$. morganii TN 373 and $P$. aeruginosa $U 31$ infections.
\end{abstract}

Expansion of the antibacterial spectrum, prevention of superinfection, and enhancement of antibacterial activity in the treatment of overwhelming infections can be expected by use of a combination of antimicrobial agents. Enhancement of antibacterial activity may also result in a reduction in the dose required for therapy and a reduction in the incidence and the severity of adverse reactions.

Cefsulodin and mecillinam have unique antibacterial spectra. The former, a cephalosporin, is active against Pseudomonas aeruginsa and some Gram-positive cocci ${ }^{1 \sim 3)}$ and the latter, a penicillin, is active against Escherichia coli and Klebsiella pneumoniae ${ }^{4 \sim 7)}$. The two antibiotics have a different mode of antibacterial mechanism. Cefsulodin binds to penicillin-binding protein (PBP) 3 in $P$. aeruginosa ${ }^{8)}$ and mecillinam binds to PBP 2 in $E$. coli ${ }^{\ominus)}$. Treatment of $P$. aeruginosa infections by cefsulodin has produced a high incidence of eradication of $P$. aeruginosa in various clinical specimens from patients, but in some cases, other bacterial species such as Serratia marcescens, Enterobacter cloacae and Proteus sp. make an appearance in place of $P$. aeruginos $a^{10)}$. Mecillinam has been used in the therapy of various kinds of urinary tract infections caused by mecillinam-susceptible organisms ${ }^{11,12}$.

These findings suggest that studies on the effect of a combination of cefsulodin and mecillinam would be of interest. The present paper examines the combination of cefsulodin and mecillinam on in vitro and in vivo activities against a number of Gram-positive and Gram-negative bacteria.

\section{Materials and Methods}

Antibiotics

Cefsulodin and mecillinam were prepared in Takeda Chemical Industries, Ltd., Osaka, Japan.

Organisms

The laboratory strains were maintained on Trypticase soy agar (TSA; BBL Microbiology Systems, Cockeysville, Md., U.S.A.). Clinical isolates were kindly supplied by several clinical laboratories and maintained on Dorset egg medium (Nissui Seiyaku Co. Ltd., Tokyo).

\section{In Vitro Test}

The minimum inhibitory concentration (MIC) was determined by the agar dilution method. The bacteria were cultured overnight at $37^{\circ} \mathrm{C}$ in Trypticase soy broth (TSB; BBL Microbiology Systems, Co- 
ckeysville, Md., U.S.A.). One loopful ( $2 \mathrm{~mm}$ in diameter) of a suspension containing about $10^{6}$ and $10^{8}$ colony-forming units (CFU)/ml was streaked for a length of about $2 \mathrm{~cm}$ on TSA or MACCONKEY agar (Eiken Chemicals, Tokyo) containing twofold serial dilutions of either or both antibiotics. The MIC was defined as the lowest concentration of antibiotic that prevented visible growth after overnight incubation at $37^{\circ} \mathrm{C}$. The effect of the combination of the two antibiotics was tested by the checkerboard dilution method ${ }^{13)}$ and the fixed combination method ${ }^{14)}$. In the checkerboard dilution method, each test consisted of 80 to 150 plates arranged horizontally and vertically, each containing different concentrations of the cefsulodin-mecillinam combination or each antibiotic alone. Eight to 10 rows in one direction contained twofold serial dilutions of cefsulodin, and 10 15 rows in the other direction contained twofold serial dilutions of mecillinam. Two additional rows contained twofold serial dilutions of cefsulodin or mecillinam only. In the fixed combination method, cefsulodin and mecillinam were combined in the ratio of 5:1 and 10:1 and the mixture of the two antibiotics was twofold serially diluted. The ratio of the two antibiotics was determined by the doses of both antibiotics used clinically. Killing kinetic studies were also performed. An overnight culture of each bacteria in TSB was inoculated into fresh TSB to yield a bacterial concentration of about $10^{4} \mathrm{CFU} / \mathrm{ml}$, and the broth was incubated with shaking at $37^{\circ} \mathrm{C}$ for $1.5 \sim 2$ hours. A $9 \mathrm{ml}$ portion of the culture, which reached about $10^{5} \mathrm{CFU} / \mathrm{ml}$ of bacterial concentration, was divided into each flask. A dose of $0.5 \mathrm{ml}$ of each antibiotic solution, prepared in the appropriate concentration, was added to the culture and when the activity of a single antibiotic was determined, $0.5 \mathrm{ml}$ of distilled water was added as a substitute for the second antibiotic. Aliquots of the culture were removed at various time intervals and the number of CFU was determined by the plate count method ${ }^{15)}$.

\section{In Vivo Test}

Four-week old male Slc: ICR mice, weighing 19 23 g, (Shizuoka Agricultural Cooperative Association for Laboratory Animals, Shizuoka, Japan) were used. Organisms were cultured in brain heart infusion (BHI; Difco Laboratories, Detroit, Mich., U.S.A.) overnight at $37^{\circ} \mathrm{C}$ and suspended in $5 \%$ mucin (Difco Laboratories, Detroit, Mich., U.S.A.). Mice were infected intraperitoneally with $0.5 \mathrm{ml}$ of the bacterial suspension. The challenge dose for each infection was about $30 \sim 100$ times the number of organisms required to kill one half of the challenged, non-treated mice. In the protection test, groups of five mice for each dose level were given $0.2 \mathrm{ml}$ of antibiotic solution subcutaneously immediately after infection. All experiments were repeated at least 5 times. The $50 \%$ effective dose $\left(\mathrm{ED}_{50}: \mathrm{mg} / \mathrm{kg}\right) \mathrm{was}$ calculated by the probit method from the survival rate, recorded 5 days after infection ${ }^{18)}$. The effect of the combination of cefsulodin and mecillinam was tested by the checkerboard dilution method and the fixed combination method at the combination ratios of cefsulodin to mecillinam of 5: 1 and 10: 1 . The number of colony-forming units (CFU) in the peritoneal washing and blood was examined in mice infected with $S$. marcescens TN 66. Blood was collected from the axillary vein and artery under ether anesthesia at various times after infection. After bleeding, $2 \mathrm{ml}$ of TSB was injected into the peritoneal cavity, the external abdominal wall gently massaged for $20 \sim 30$ seconds and the peritoneal washing collected. The blood and peritoneal washing were serially 10 -fold diluted with distilled water and $0.1 \mathrm{ml}$ of the sample was inoculated onto a TSA plate. The plates were incubated overnight at $37^{\circ} \mathrm{C}$ and the colonies were counted. Bacterial counts were expressed as the number of CFU per ml of the blood and peritoneal washing.

\section{Criteria for the Combination Effect}

To compare the effect of cefsulodin plus mecillinam with the two antibiotics individually the fractional inhibitory concentration (FIC) and FIC index were calculated according to the method reported by ElION et al. ${ }^{17)} \quad$ Criteria for the effect of combination were defined as follows: 1) Synergy-the FIC index is lower than 0.5. 2) Addition-the FIC index is $0.5 \sim 2.0$. 3) Antagonism-the FIC index is higher than 2.0. 4) Indifferent-with the checkerboard dilution method, the MIC of either antibiotic is more than $100 \mu \mathrm{g} / \mathrm{ml}$ and the $\mathrm{MIC}$ value of the other is not reduced by the combination. In the fixed combination method, the MICs of both antibiotics are more than $100 \mu \mathrm{g} / \mathrm{ml}$. For the calculation of the FIC value, when the growth of an organism is not inhibited at a concentration of $100 \mu \mathrm{g} / \mathrm{ml}$ of either antibiotic, the MIC is assessed as $200 \mu \mathrm{g} / \mathrm{ml}$. On the killing kinetics, the following definitions were 
used for the combination effect ${ }^{18)}$. 1) Synergy - the CFU declines 100 times or more from that occurring with either antibiotic alone. 2) Addition-the CFU is reduced to one-tenth or less than that occurring with either antibiotic alone. 3) Antagonism - the CFU increases 10 times or more than that occurring with either antibiotic alone. 4) Indifferent- the CFU is within 10 times that produced by either antibiotic alone. To assess the protective effect of the combination of the two antibiotics, the fractional effective dose (FED) and FED index were used as a substitute for the FIC and FIC index.

\section{Results}

In Vitro Test

Antibacterial Spectrum

Cefsulodin showed potent antibacterial activity against $S$. aureus and $P$. aeruginosa. The antibacterial activity of mecillinam was potent against E. coli, K. pneumoniae and Serratia marcescens. By the combination of cefsulodin and mecillinam in the ratio of 5:1 and 10:1, the antibacterial spectrum was wider than that of each antibiotic alone and this was more obvious with the inoculum size of $10^{6}$ $\mathrm{CFU} / \mathrm{ml}$ (Table 1). The mixture of both antibiotics was active against Citrobacter freundii, Enterobacter cloacae and Proteus sp. which were not affected by each antibiotic alone.

\section{Checkerboard-combination}

The FIC and FIC index values of the combination of cefsulodin and mecillinam using the checkerboard dilution method on 26 strains of 14 bacterial species are summarized in Table 2 and a typical isobolograms showing synergy of these antibiotics against S. marcescens IFO 12648 and TN 66 are shown in Fig. 1. A synergistic effect (FIC index; lower than 0.5) was observed on both strains of C. freundii, E. cloacae and S. marcescens, and one of two strains of Proteus mirabilis, Proteus vulgaris and Proteus inconstans. In particular, the MIC values of cefsulodin and mecillinam against all strains of C. freundii, E. cloacae and $S$. marcescens decreased 16 64 times with the combination of both antibiotics. An additive antibacterial effect (FIC index; $0.5 \sim 2.0$ ) was observed on 17 out of 27 strains tested. An

Table 1. Antibacterial spectrum of cefsulodin, mecillinam and a mixture of the two antibiotics.

\begin{tabular}{|c|c|c|c|c|c|c|c|c|}
\hline \multirow{3}{*}{ Organism } & \multicolumn{4}{|c|}{$\left.10^{8} \mathrm{CFU} / \mathrm{ml}^{\mathrm{a}}\right)$} & \multicolumn{4}{|c|}{$10^{6} \mathrm{CFU} / \mathrm{ml}$} \\
\hline & \multirow{2}{*}{$\begin{array}{c}\text { Cef- } \\
\text { sulodin }\end{array}$} & \multirow{2}{*}{$\begin{array}{l}\text { Mecil- } \\
\text { linam }\end{array}$} & \multicolumn{2}{|c|}{$\begin{array}{c}\text { Cef- } \\
\text { sulodin }\end{array}+\begin{array}{l}\text { Mecil- } \\
\text { linam }\end{array}$} & \multirow{2}{*}{$\begin{array}{c}\text { Cef- } \\
\text { sulodin }\end{array}$} & \multirow{2}{*}{$\begin{array}{l}\text { Mecil- } \\
\text { linam }\end{array}$} & \multicolumn{2}{|c|}{$\begin{array}{c}\text { Cef- } \\
\text { sulodin }\end{array}+\begin{array}{l}\text { Mecil- } \\
\text { linam }\end{array}$} \\
\hline & & & $5: 1$ & $10: 1$ & & & $5: 1$ & $10: 1$ \\
\hline Staphylococcus aureus FDA 209P & 6.25 & 25 & 6.25 & 6.25 & 3.13 & 12.5 & 3.13 & 3.13 \\
\hline $\begin{array}{ll}\prime \prime & 308 \mathrm{~A}-1\end{array}$ & 3.13 & 25 & 3.13 & 3.13 & 3.13 & 12.5 & 3.13 & 3.13 \\
\hline Escherichia coli NIHJ JC-2 & 50 & 0.2 & 1.56 & 3.13 & 50 & 0.2 & 0.78 & 1.56 \\
\hline " $\quad \mathrm{O}-111$ & 50 & 0.1 & 0.78 & 0.78 & 50 & 0.05 & 0.39 & 0.39 \\
\hline Citrobacter freundii IFO 12681 & 50 & $>100$ & 6.25 & 6.25 & 50 & 100 & 3.13 & 1.56 \\
\hline Klebsiella pneumoniae DT & 50 & 50 & 6.25 & 6.25 & 50 & 0.1 & 0.78 & 0.78 \\
\hline Enterobacter cloacae IFO 12937 & $>100$ & $>100$ & 25 & 25 & $>100$ & 25 & 3.13 & 6.25 \\
\hline Serratia marcescens IFO 12648 & 100 & $>100$ & 12.5 & 12.5 & 100 & 1.56 & 6.25 & 6.25 \\
\hline Proteus mirabilis IFO 3849 & 100 & 100 & 25 & 25 & 100 & 25 & 6.25 & 12.5 \\
\hline Proteus vulgaris IFO 3988 & 100 & $>100$ & 25 & 25 & 100 & 50 & 12.5 & 12.5 \\
\hline Proteus morganii IFO 3168 & $>100$ & $>100$ & 100 & 50 & $>100$ & $>100$ & 50 & 50 \\
\hline Pseudomonas aeruginosa U 31 & 12.5 & $>100$ & 12.5 & 12.5 & 1.56 & $>100$ & 3.13 & 3.13 \\
\hline
\end{tabular}

a) MIC $(\mu \mathrm{g} / \mathrm{ml})$ was determined on TSA. Inoculum size was one loopful of bacterial suspension. 
Table 2. Effect of combination of cefsulodin and mecillinam on in vitro antibacterial activity with the checkerboard dilution method.

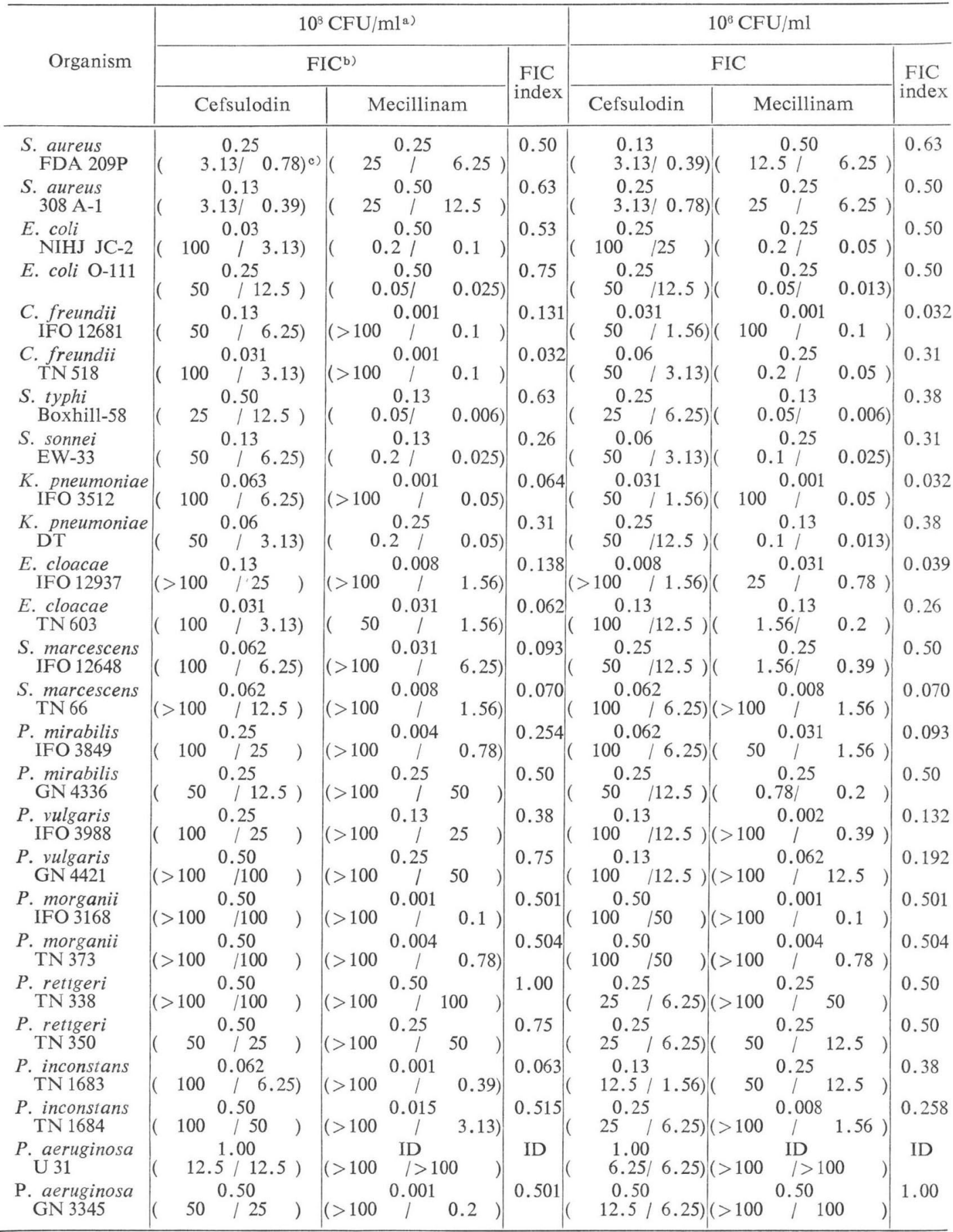

a) Inoculum size: One loopful of bacterial suspension.

b) Fractional Inhibitory Concentration.

e) Number in parentheses indicates MIC of first cephalosporin alone/MIC of first cephalosporin in combination of second cephalosporin. 
Fig. 1. Isoborogram showing synergy of cefsulodin and mecillinam against $S$. marcescens with the inoculum sizes of $10^{8} \mathrm{CFU} / \mathrm{ml}$ and $10^{6} \mathrm{CFU} / \mathrm{ml}$.

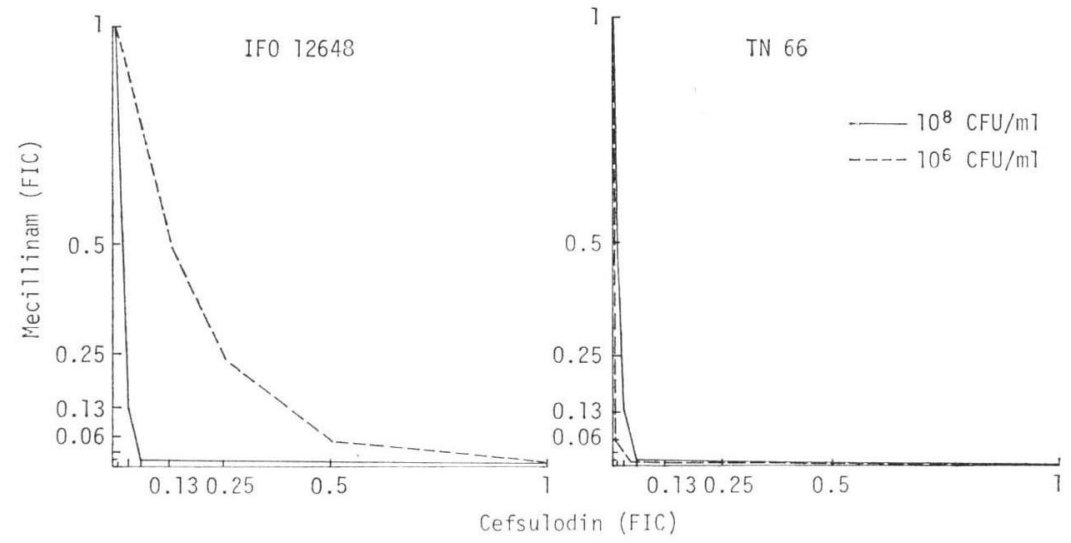

Table 3. Effect of combination of cefsulodin and mecillinam against clinical isolates with the fixed combination method.

\begin{tabular}{|c|c|c|c|c|c|c|c|c|c|}
\hline \multirow{3}{*}{$\begin{array}{l}\text { Organism } \\
\text { (No. of strains } \\
\text { tested) }\end{array}$} & \multirow{3}{*}{$\begin{array}{c}\text { Combination } \\
\text { ratio } \\
\text { (Cefsulodin } \\
\text { vs. } \\
\text { mecillinam) }\end{array}$} & \multicolumn{8}{|c|}{ No. of strains exhibiting } \\
\hline & & \multicolumn{4}{|c|}{$10^{\mathrm{s}} \mathrm{CFU} / \mathrm{ml}^{\mathrm{a})}$} & \multicolumn{4}{|c|}{$10^{6} \mathrm{CFU} / \mathrm{ml}$} \\
\hline & & Synergy & $\begin{array}{l}\text { Addi- } \\
\text { tion }\end{array}$ & $\begin{array}{l}\text { Antag- } \\
\text { onism }\end{array}$ & $\begin{array}{l}\text { Indif- } \\
\text { ference }\end{array}$ & Synergy & $\begin{array}{l}\text { Addi- } \\
\text { tion }\end{array}$ & $\begin{array}{l}\text { Antag- } \\
\text { onism }\end{array}$ & $\begin{array}{l}\text { Indif- } \\
\text { ference }\end{array}$ \\
\hline $\begin{array}{l}\text { S. aureus } \\
(38)\end{array}$ & $\begin{array}{r}5: 1 \\
10: 1\end{array}$ & & $\begin{array}{l}38 \\
38\end{array}$ & & & 1 & $\begin{array}{l}38 \\
37\end{array}$ & & \\
\hline E. coli & $\begin{array}{r}5: 1 \\
10: 1\end{array}$ & $\begin{array}{l}35 \\
34\end{array}$ & $\begin{array}{l}2 \\
3\end{array}$ & & & $\begin{array}{l}6 \\
6\end{array}$ & $\begin{array}{l}31 \\
31\end{array}$ & & \\
\hline C. freundii & $\begin{array}{r}5: 1 \\
10: 1\end{array}$ & $\begin{array}{l}32 \\
30\end{array}$ & $\begin{array}{l}1 \\
3\end{array}$ & & $\begin{array}{l}5 \\
5\end{array}$ & $\begin{array}{r}7 \\
12\end{array}$ & $\begin{array}{l}29 \\
23\end{array}$ & & $\begin{array}{l}2 \\
3\end{array}$ \\
\hline$K . \underset{(37)}{\text { pneumoniae }}$ & $\begin{array}{r}5: 1 \\
10: 1\end{array}$ & $\begin{array}{l}30 \\
32\end{array}$ & $\begin{array}{l}6 \\
4\end{array}$ & & $\begin{array}{l}1 \\
1\end{array}$ & $\begin{array}{l}19 \\
19\end{array}$ & $\begin{array}{l}18 \\
17\end{array}$ & 1 & 1 \\
\hline $\begin{array}{r}\text { E. cloacae } \\
(38)\end{array}$ & $\begin{array}{r}5: 1 \\
10: 1\end{array}$ & $\begin{array}{l}32 \\
31\end{array}$ & $\begin{array}{l}2 \\
3\end{array}$ & & $\begin{array}{l}4 \\
4\end{array}$ & $\begin{array}{l}13 \\
15\end{array}$ & $\begin{array}{l}24 \\
21\end{array}$ & & $\begin{array}{l}1 \\
2\end{array}$ \\
\hline $\begin{array}{c}\text { S. marcescens } \\
(36)\end{array}$ & $\begin{array}{r}5: 1 \\
10: 1\end{array}$ & $\begin{array}{l}27 \\
27\end{array}$ & $\begin{array}{l}2 \\
2\end{array}$ & & $\begin{array}{l}7 \\
7\end{array}$ & $\begin{array}{l}31 \\
29\end{array}$ & $\begin{array}{l}2 \\
2\end{array}$ & & $\begin{array}{l}3 \\
5\end{array}$ \\
\hline $\begin{array}{l}\text { P. mirabilis } \\
(38)\end{array}$ & $\begin{array}{r}5: 1 \\
10: 1\end{array}$ & $\begin{array}{l}32 \\
34\end{array}$ & 1 & & $\begin{array}{l}5 \\
4\end{array}$ & $\begin{array}{l}33 \\
37\end{array}$ & $\begin{array}{l}5 \\
1\end{array}$ & & \\
\hline P. vulgaris & $\begin{array}{r}5: 1 \\
10: 1\end{array}$ & $\begin{array}{l}20 \\
23\end{array}$ & $\begin{array}{l}2 \\
2\end{array}$ & & $\begin{array}{l}16 \\
13\end{array}$ & $\begin{array}{l}22 \\
22\end{array}$ & $\begin{array}{l}13 \\
13\end{array}$ & $\begin{array}{l}1 \\
1\end{array}$ & $\frac{2}{2}$ \\
\hline P. $\underset{(37)}{\operatorname{morganii}}$ & $\begin{array}{r}5: 1 \\
10: 1\end{array}$ & $\begin{array}{l}18 \\
18\end{array}$ & $\begin{array}{l}19 \\
17\end{array}$ & 2 & & $\begin{array}{l}17 \\
16\end{array}$ & $\begin{array}{l}18 \\
20\end{array}$ & $\begin{array}{l}2 \\
1\end{array}$ & \\
\hline $\begin{array}{c}\text { P. rettgeri } \\
(38)\end{array}$ & $\begin{array}{r}5: 1 \\
10: 1\end{array}$ & $\begin{array}{l}18 \\
18\end{array}$ & $\begin{array}{l}18 \\
18\end{array}$ & & $\begin{array}{l}2 \\
2\end{array}$ & $\begin{array}{l}21 \\
21\end{array}$ & $\begin{array}{l}15 \\
15\end{array}$ & & $\begin{array}{l}2 \\
2\end{array}$ \\
\hline $\begin{array}{c}\text { P. inconstans } \\
(27)\end{array}$ & $\begin{array}{r}5: 1 \\
10: 1\end{array}$ & $\begin{array}{l}21 \\
21\end{array}$ & $\begin{array}{l}4 \\
4\end{array}$ & & $\begin{array}{l}2 \\
2\end{array}$ & $\begin{array}{l}17 \\
18\end{array}$ & $\begin{array}{r}10 \\
9\end{array}$ & & \\
\hline $\begin{array}{l}P \text { aeruginosa } \\
\text { (37) }\end{array}$ & $\begin{array}{r}5: 1 \\
10: 1\end{array}$ & 1 & $\begin{array}{l}36 \\
37\end{array}$ & & & 5 & $\begin{array}{l}37 \\
32\end{array}$ & & \\
\hline
\end{tabular}

a) Inoculum size was one loopful of bacterial suspension.

antagonistic effect (FIC index; more than 2.0) was not observed on any of the bacterial strains tested. With the combination of cefsulodin and mecillinam, and mecillinam alone, $P$. aeruginosa U 31 was not inhibited at a concentration of $100 \mu \mathrm{g} / \mathrm{ml}$ : this strain was classified as indifferent. 
Fixed-combination

Cefsulodin and mecillinam alone and the fixed combination of the two antibiotics in the ratio of $5: 1$ and 10: 1 against clinical isolates were examined and the combined effects are summarized in Table 3 . With the inoculum size of $10^{8} \mathrm{CFU} / \mathrm{ml}$, a synergistic effect was observed on most strains of E. coli, C. freundii, K. pneumoniae, E. cloacae, S. marcescens, $P$. mirabilis, $P$. vulgaris, $P$. inconstans and on about one half of the strains of $P$. morganii and $P$. rettgeri. With the inoculum size of $10^{\circ} \mathrm{CFU} / \mathrm{ml}$, the synergistic effect was observed on most strains of $S$. marcescens, $P$. mirabilis, $P$. vulgaris, and $P$. inconstans, and on about one half of the strains of K. pneumoniae, E. cloacae, and P. morganii. An antagonistic effect was observed on only a few strains of $K$. pneumoniae, $P$. vulgaris, and $P$. morganii. An indifferent effect was observed on some strains of $C$. freundii, K. pneumoniae, E. cloacae, S. marcescens, P. mirabilis, $P$. vulgaris, $P$. rettgeri, and $P$. inconstans. On the remaining strains of various bacterial species, especially on almost all strains of $S$. aureus and $P$. aeruginosa, the effect of combination of cefsulodin and mecillinam was additional.

\section{Killing Kinetic}

The bactericidal activity of the combination of cefsulodin and mecillinam compared with that of either of the antibiotics is summarized in Table 4 and as a typical picture of the killing kinetics test, the killing curves of the two antibiotics against S. marcescens IFO 12648 and TN 66 are shown in Fig. 2. A synergistic effect was observed on E. coli NIHJ JC-2, C. freundii TN 518, K. pneumoniae DT, E. cloacae IFO 12937, S. marcescens IFO 12648 and TN 66, P. mirabilis IFO 3849 and P. vulgaris IFO 3988. The number of CFU of these strains was not decreased at the concentrations of each antibiotic used alone and

Table 4. Effect of combination of cefsulodin and mecillinam against 11 strains of bacteria with the killing kinetic method.

\begin{tabular}{|c|c|c|c|c|}
\hline \multirow{2}{*}{ Organism } & \multicolumn{2}{|c|}{ Antibiotic $(\mu \mathrm{g} / \mathrm{ml})$} & \multirow{2}{*}{$\begin{array}{l}\text { Rate of changea) } \\
\text { of No. of CFU }\end{array}$} & \multirow{2}{*}{$\begin{array}{l}\text { Effect of } \\
\text { combination }\end{array}$} \\
\hline & Cefsulodin & + Mecillinam & & \\
\hline S. aureus FDA 209P & $\begin{array}{l}0.78 \\
1.56\end{array}$ & $\begin{array}{l}6.25 \\
6.25\end{array}$ & $\begin{array}{l}2 \times 10^{-2} \\
2 \times 10^{-4}\end{array}$ & $\begin{array}{l}\text { Synergy } \\
\text { Synergy }\end{array}$ \\
\hline E. coli NIHJ JC-2 & $\begin{array}{l}12.5 \\
25\end{array}$ & $\begin{array}{l}0.1 \\
0.1\end{array}$ & $\begin{array}{l}8 \times 10^{-4} \\
3 \times 10^{-6}\end{array}$ & $\begin{array}{l}\text { Synergy } \\
\text { Synergy }\end{array}$ \\
\hline C. freundii TN 518 & $\frac{12.5}{25}$ & $\begin{array}{l}0.1 \\
0.1\end{array}$ & $\begin{array}{l}9 \times 10^{-5} \\
8 \times 10^{-5}\end{array}$ & $\begin{array}{l}\text { Synergy } \\
\text { Synergy }\end{array}$ \\
\hline K. pneumoniae DT & 12.5 & $\begin{array}{l}0.05 \\
0.05\end{array}$ & $\begin{array}{l}6 \times 10^{-4} \\
2 \times 10^{-3}\end{array}$ & $\begin{array}{l}\text { Synergy } \\
\text { Synergy }\end{array}$ \\
\hline E. cloacae IFO 12937 & $\frac{12.5}{25}$ & $\begin{array}{l}0.39 \\
0.39\end{array}$ & $\begin{array}{l}2 \times 10^{-4} \\
2 \times 10^{-4}\end{array}$ & $\begin{array}{l}\text { Synergy } \\
\text { Synergy }\end{array}$ \\
\hline S. marcescens IFO 12648 & $\begin{array}{l}12.5 \\
25\end{array}$ & $\begin{array}{l}0.2 \\
0.2\end{array}$ & $\begin{array}{l}3 \times 10^{-5} \\
2 \times 10^{-5}\end{array}$ & $\begin{array}{l}\text { Synergy } \\
\text { Synergy }\end{array}$ \\
\hline S. marcescens TN 66 & $\frac{12.5}{25}$ & $\begin{array}{l}0.78 \\
0.78\end{array}$ & $\begin{array}{l}3 \times 10^{-3} \\
3 \times 10^{-3}\end{array}$ & $\begin{array}{l}\text { Synergy } \\
\text { Synergy }\end{array}$ \\
\hline P. mirabilis IFO 3849 & $\frac{12.5}{25}$ & $\begin{array}{l}1.56 \\
1.56\end{array}$ & $\begin{array}{l}6 \times 10^{-4} \\
2 \times 10^{-4}\end{array}$ & $\begin{array}{l}\text { Synergy } \\
\text { Synergy }\end{array}$ \\
\hline P. vulgaris IFO 3988 & $\begin{array}{l}12.5 \\
25\end{array}$ & $\begin{array}{l}0.2 \\
0.2\end{array}$ & $\begin{array}{l}7 \times 10^{-3} \\
6 \times 10^{-5}\end{array}$ & $\begin{array}{l}\text { Synergy } \\
\text { Synergy }\end{array}$ \\
\hline P. morganii TN 373 & $\begin{array}{l}12.5 \\
25\end{array}$ & $\begin{array}{l}6.25 \\
6.25\end{array}$ & $\begin{array}{l}4 \times 10^{-1} \\
4 \times 10^{-1}\end{array}$ & $\begin{array}{l}\text { Addition } \\
\text { Addition }\end{array}$ \\
\hline P. aeruginosa U 31 & $\begin{array}{l}1.56 \\
3.13\end{array}$ & $\begin{array}{l}400 \\
400\end{array}$ & $\begin{array}{l}2 \times 10^{-1} \\
1 \times 10^{1}\end{array}$ & $\begin{array}{l}\text { Addition } \\
\text { Indifferent }\end{array}$ \\
\hline
\end{tabular}

a) The number of CFU by combination of antibiotics/the number of CFU by an antibiotic alone. 
Fig. 2. Killing curve showing synergy of cefsulodin (CFS) and mecillinam (MPC) against S. marcescens.

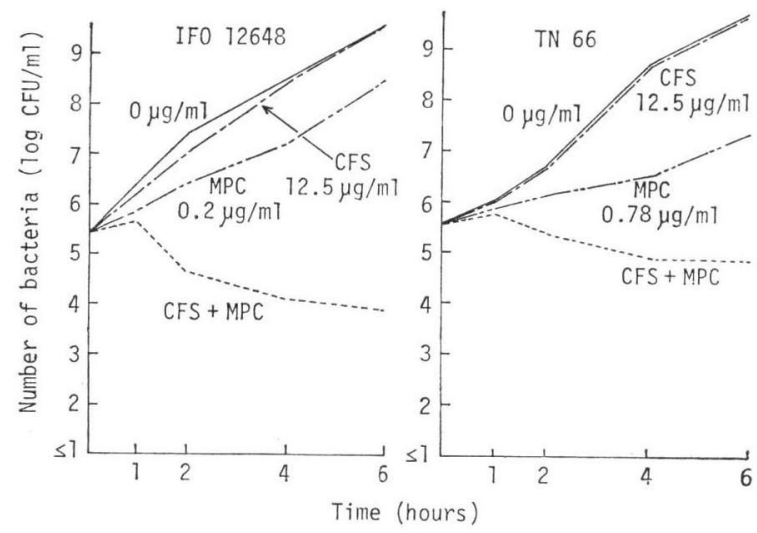

decreased more than 100 times with the combination of cefsulodin and mecillinam. The effect of the combination against $P$. morganii TN 373 and $P$. aeruginosa $\mathrm{U} 31$ was classified as additional or indifferent, since the number of CFU of these strains was slightly decreased or increased with the combination of both antibiotics compared with either antibiotic alone.

\section{In Vivo Test}

\section{Synergistic Effect against $S$. marcescens TN 66}

In in vitro studies, cefsulodin and mecillinam showed a marked synergistic effect against several laboratory and clinical strains of S. marcescens. S. marcescens TN 66 was selected as the in vivo test organism, since the strain shows potent virulence on intraperitoneal infection with mucin. A synergistic effect of the combination of cefsulodin and mecillinam was observed in the protective test in mice using the checkerboard dilution method. The survival rates of mice treated with either or both antibiotics are shown in Table 5 and the $\mathrm{ED}_{50}$ values and FED index calculated from the results presented in Table 5 are

Table 5. Survival rate of mice infected with $S$. marcescens TN 66 treated by a combination of cefsulodin plus mecillinam. ${ }^{\text {a) }}$

\begin{tabular}{c|c|c|c|c|c|c|c}
\hline \multirow{2}{*}{$\begin{array}{c}\text { Mecillinam } \\
(\mathrm{mg} / \mathrm{kg})\end{array}$} & \multicolumn{7}{|c}{ Cefsulodin (mg/kg) } \\
\cline { 2 - 7 } & 0 & 6.25 & 12.5 & 25 & 50 & 100 & 200 \\
\hline 0 & $0 / 25^{\mathrm{b})}$ & $0 / 25$ & $0 / 25$ & $0 / 25$ & $0 / 25$ & $10 / 25$ & $20 / 25$ \\
1.56 & & & $0 / 25$ & $0 / 25$ & $4 / 25$ & $22 / 25$ & $25 / 25$ \\
3.13 & & & $0 / 25$ & $3 / 25$ & $4 / 25$ & $22 / 25$ & $25 / 25$ \\
6.25 & & & $0 / 25$ & $2 / 25$ & $6 / 25$ & $20 / 25$ & $25 / 25$ \\
12.5 & & & $0 / 25$ & $4 / 25$ & $12 / 25$ & $25 / 25$ & $25 / 25$ \\
25 & $0 / 25$ & $0 / 25$ & $3 / 25$ & $6 / 25$ & $11 / 25$ & $25 / 25$ & $25 / 25$ \\
50 & $0 / 25$ & $0 / 25$ & $7 / 25$ & $10 / 25$ & $11 / 25$ & $25 / 25$ & $25 / 25$ \\
100 & $0 / 25$ & $0 / 25$ & $6 / 25$ & $10 / 25$ & $16 / 25$ & $25 / 25$ & $25 / 25$ \\
200 & $4 / 25$ & $5 / 25$ & $10 / 25$ & $14 / 25$ & $14 / 25$ & $25 / 25$ & $25 / 25$ \\
400 & $8 / 25$ & $10 / 25$ & $15 / 25$ & $22 / 25$ & $22 / 25$ & $25 / 25$ & $25 / 25$ \\
800 & $15 / 25$ & $20 / 25$ & $24 / 25$ & $25 / 25$ & $25 / 25$ & $25 / 25$ & $25 / 25$ \\
\hline
\end{tabular}

a) Mice were infected intraperitoneally with $S$. marcescens TN $66\left(10^{3} \mathrm{CFU} / \mathrm{mouse}\right)$ in $0.5 \mathrm{ml}$ of $5 \%$ mucin. Antibiotics were administered subcutaneously at 0 hour after infection.

b) No. of survival mice/No. of total mice. 
Table 6. Effect of combination of cefsulodin and mecillinam on mice infected with $S$. marcescens TN 66. ${ }^{a)}$

\begin{tabular}{|c|c|c|c|c|c|c|c|c|c|}
\hline \multicolumn{3}{|c|}{ Ratio } & \multirow{2}{*}{\multicolumn{4}{|c|}{$\begin{array}{c}\mathrm{ED}_{50} ; \mathrm{mg} / \mathrm{kg} \\
(95 \% \text { confidence limit })\end{array}$}} & \multicolumn{2}{|c|}{ FED $\left.^{b}\right)$} & \multirow{2}{*}{ FED Index } \\
\hline Cefsulodin & & Mecillinam & & & & & \multirow[t]{2}{*}{ Cefsulodin } & \multirow[t]{3}{*}{ Mecillinam } & \\
\hline Alone & & - & 124 & $(103$ & $\sim$ & 149 ) & & & \\
\hline- & & Alone & 613 & $(475$ & $\sim 1$ & $, 000 \quad)$ & & & \\
\hline 32 & : & 1 & 71.6 & $(60$. & & $83.6)$ & $\begin{array}{c}0.560 \\
(69.4 / 124)\end{array}$ & $\begin{array}{ll} & 0.004 \\
( & 2.2 / 613)\end{array}$ & 0.564 \\
\hline 16 & : & 1 & 77.2 & ( 65. & $4 \sim$ & 91.1) & $\begin{array}{c}0.586 \\
(72.7 / 124)\end{array}$ & $\begin{array}{l}0.007 \\
(4.5 / 613)\end{array}$ & 0.593 \\
\hline 8 & : & 1 & 65.9 & $(57$. & $7 \sim$ & $76.5)$ & $\begin{array}{c}0.473 \\
(58.6 / 124)\end{array}$ & $\begin{array}{ll} & 0.012 \\
(\quad 7.3 / 613)\end{array}$ & 0.485 \\
\hline 4 & : & 1 & 59.6 & $(50$. & $4 \sim$ & $70.3)$ & $\begin{array}{c}0.385 \\
(47.4 / 124)\end{array}$ & $\begin{array}{c}0.019 \\
(11.9 / 613)\end{array}$ & 0.404 \\
\hline 2 & : & 1 & 69.1 & ( 57 . & $1 \sim$ & $83.0)$ & $\begin{array}{c}0.372 \\
(46.1 / 124)\end{array}$ & $\begin{array}{c}0.038 \\
(23.0 / 613)\end{array}$ & 0.410 \\
\hline 1 & : & 1 & 88.7 & ( 70 . & $2 \sim$ & $110)$ & $\begin{array}{c}0.358 \\
(44.4 / 124)\end{array}$ & $\begin{array}{c}0.072 \\
(44.4 / 613)\end{array}$ & 0.430 \\
\hline 1 & : & 2 & 94.1 & ( 74 . & $4 \sim$ & 118 ) & $\begin{array}{c}0.253 \\
(31.4 / 124)\end{array}$ & $\begin{array}{c}0.102 \\
(62.7 / 613)\end{array}$ & 0.355 \\
\hline 1 & : & 4 & 150 & ( 60. & $3 \sim$ & $444 \quad)$ & $\begin{array}{c}0.242 \\
(30.0 / 124)\end{array}$ & $\begin{array}{c}0.196 \\
\left(120^{/ 613}\right)\end{array}$ & 0.438 \\
\hline 1 & : & 8 & 199 & (162 & $\sim$ & 245 ) & $\begin{array}{c}0.177 \\
(22.0 / 124)\end{array}$ & $\begin{array}{c}0.289 \\
(177 / 613)\end{array}$ & 0.466 \\
\hline 1 & : & 16 & 250 & $(210$ & $\sim$ & 297 ) & $\begin{array}{c}0.121 \\
(15.0 / 124)\end{array}$ & $\begin{array}{c}0.383 \\
(235 \quad / 613)\end{array}$ & 0.504 \\
\hline 1 & : & 32 & 332 & $(272$ & $\sim$ & $399 \quad$ & $\begin{array}{c}0.081 \\
(10.0 / 124)\end{array}$ & $\begin{array}{c}0.525 \\
(322 \quad / 613)\end{array}$ & 0.606 \\
\hline
\end{tabular}

a) Mice were infected intraperitoneally with $S$. marcescens TN $66\left(10^{3} \mathrm{CFU} / \mathrm{mouse}\right)$ in $0.5 \mathrm{ml}$ of $5 \%$ mucin. Antibiotics were administered subcutaneously at 0 hour after infection.

b) Number in parentheses indicates $\mathrm{ED}_{50}$ of one antibiotic in the presence of another/ED $\mathrm{ED}_{50}$ of the indicated antibiotic alone.

Fig. 3. Killing curve showing synergy of cefsulodin and mecillinam on bacterial count in peritoneal washing and blood of mice infected with S. marcescens TN 66 .

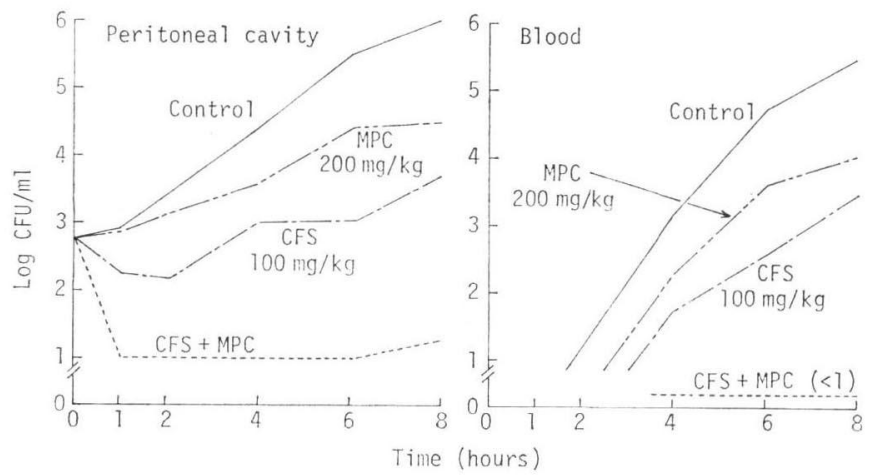

summarized in Table 6. The $\mathrm{ED}_{50}$ values of cefsulodin and mecillinam alone were $124 \mathrm{and} 613 \mathrm{mg} / \mathrm{kg}$, respectively, and with the combination of the two antibiotics, the $\mathrm{ED}_{50}$ values definitely decreased. The smallest $\mathrm{ED}_{50}$ value was observed with the ratio of cefsulodin to mecillinam of $4: 1$, but the minimum FED index, 0.355, was observed with the ratio of cefsulodin to mecillinam of $1: 2$. The number of CFU 
Table 7. Effect of combination of cefsulodin and mecillinam on intraperitoneal infection in mice with the fixed-combination method. ${ }^{\text {s }}$

\begin{tabular}{|c|c|c|c|c|c|c|}
\hline \multirow{3}{*}{$\begin{array}{c}\text { Organism } \\
\text { (CFU/mouse) }\end{array}$} & \multicolumn{4}{|c|}{$\mathrm{ED}_{50}(\mathrm{mg} / \mathrm{kg})^{\mathrm{b})}$} & \multicolumn{2}{|c|}{ FED Index } \\
\hline & \multirow{2}{*}{ Cefsulodin } & \multirow{2}{*}{ Mecillinam } & Cefsulodin & Mecillinam & Cefsulodin & + Mecillinam \\
\hline & & & $5: 1$ & $10: 1$ & $5: 1$ & $10: 1$ \\
\hline $\begin{array}{l}\text { S. aureus } \\
\underset{\left(10^{8}\right)}{\text { and }}\end{array}$ & $(\quad 2.71 \sim 46.51)$ & $(125 \stackrel{174}{\sim} 247$ & $\left(2.88^{3.75} \sim 4.93\right)$ & $\left(3.76^{4.65} \sim 5.86\right)$ & 0.91 & 1.22 \\
\hline E. coli $\mathrm{O}-111$ & $\left(31.4^{39.1} \sim 47.3\right)$ & $\left(0.035 \sim^{0.044} 0.053\right)$ & $(0.183 \sim 0.274)$ & $\begin{array}{c}0.330 \\
(0.243 \sim 0.418)\end{array}$ & 0.87 & 0.69 \\
\hline E. coli T 7 & $(222 \stackrel{268}{\sim 328 \quad})$ & $\left(7.45 \sim{ }^{9.62} 12.3\right)$ & $\left(34.7^{41.4} \sim 49.4\right)$ & $(59.1 \stackrel{70.5}{\sim} 84.1)$ & 0.85 & 0.91 \\
\hline $\begin{array}{l}\text { C. freundii } \\
\left(10^{5}\right)\end{array}$ & $(17.5 \stackrel{20.7}{\sim} 24.6)$ & $\left(\quad 0.338 \sim^{0.401} 0.473\right)$ & $(1.05 \stackrel{1.32}{\sim} 1.73)$ & $\left(1.79^{2.13} \sim 2.53\right)$ & 0.60 & 0.56 \\
\hline K. pneumoniae DT & $(26.2 \stackrel{32.4}{\sim} 39.9)$ & $\left(4.44 \sim^{6.18} 10.5\right)$ & $\left(5.48^{7.59} \sim 11.0\right)$ & $(5.97 \stackrel{7.26}{\sim} 8.84)$ & 0.40 & 0.31 \\
\hline $\begin{array}{l}\text { E. cloacae TN } 603 \\
\left(10^{4}\right)\end{array}$ & $(47.6 \stackrel{58.0}{\sim 70.7})$ & $\left(0.539 \sim^{0.738} 0.936\right)$ & $\left(1.96^{2.41} \sim 2.95\right)$ & $\left(3.06^{3.79} \sim 4.73\right)$ & 0.59 & 0.53 \\
\hline $\begin{array}{l}\text { S. marcescens } \mathrm{TN} 66 \\
\left(10^{3}\right)\end{array}$ & $\left(96.7^{118} \sim 142 \quad\right)$ & $>800$ & $\left(39.3^{48.5} \sim 59.5\right)$ & $\left(46.0^{56.3} \sim 69.0\right)$ & 0.35 & 0.44 \\
\hline P. vulgaris $\mathrm{GN} 4712$ & $(146 \stackrel{186}{\sim 247 \quad)}$ & $(39.9 \stackrel{54.3}{\sim} 78.6)$ & $\left(58.2^{75.1} \sim 100\right.$ & $\left(57.9^{73.5} \sim 93.7\right)$ & 0.57 & 0.48 \\
\hline P. morganii $\mathrm{TN} 373$ & $(45.5 \stackrel{54.1}{\sim} 64.6)$ & $\left(\begin{array}{l}6496 \\
\sim 2,740\end{array}\right.$ & $\left(24.0^{32.9} \sim 43.7\right)$ & $\left(24.9^{30.4} \sim 37.5\right)$ & 0.51 & 0.51 \\
\hline $\begin{array}{l}P . \underset{\left(10^{6}\right)}{\text { aeruginos }} \\
\mathrm{U}\end{array} 31$ & $(\quad 3.77 \sim 7.40)$ & $>800$ & $(5.40 \stackrel{7.74}{\sim} 10.4)$ & $\left(4.14^{6.01} \sim 7.95\right)$ & 1.19 & 1.01 \\
\hline
\end{tabular}

a) Mice were infected intraperitoneally with test organism in $0.5 \mathrm{ml}$ of $5 \%$ mucin

b) Antibiotics were administered subcutaneously at 0 hour after infection.

$\mathrm{ED}_{50}$ values were calculated by the probit method. Number in parentheses indicates $95 \%$ confidence limits. 
in the peritoneal washing and blood of mice treated with $100 \mathrm{mg}$ of cefsulodin per $\mathrm{kg}$ and $200 \mathrm{mg}$ of mecillinam per $\mathrm{kg}$ was compared with that of each antibiotic alone (Fig. 3). In mice treated with cefsulodin or mecillinam alone, the increase in the number of CFU in the peritoneal washing and blood was greater than that in the control mice. By the combination of the two antibiotics, the number of CFU in the peritoneal washing decreased to about $10 \mathrm{CFU} / \mathrm{ml}$ at 1 hour after treatment and the same level was maintained for up to 8 hours. No bacterial count could be made in the blood of mice treated with the combination of cefsulodin and mecillinam.

Fixed-combination

The effect of the combination of cefsulodin and mecillinam in the ratio of $5: 1$ and 10:1 in mice infected intraperitoneally with 10 bacterial strains in 9 species is shown in Table 7. A synergistic effect (FED index; lower than 0.5) was observed in mice infected with $K$. pneumoniae DT and $S$. marcescens TN 66. In mice infected with other test bacterial strains, the FED index ranged from about $0.5 \sim 1.2$ and these findings indicate that the combination of the two antibiotics resulted in an additive effect.

\section{Discussion}

The combination of cefsulodin with other antibiotics is of potential clinical interest because cefsulodin has a very narrow antibacterial spectrum. A potent antibacterial activity of cefsulodin has been observed on $P$. aeruginosa and $S$. aureus and the activity against other Gram-negative rods is weak. The mode of action of cefsulodin against $P$. aeruginosa is different from that of many $\beta$-lactam antibiotics against Gram-negative rods but that against $E$. coli, which showed weak susceptibility, was similar to that of $\beta$-lactam antibiotics ${ }^{8}$. Mecillinam has an antibacterial activity against certain Gram-negative rods $^{4 \sim 7)}$ and shows a unique mode of action ${ }^{9)}$. In the present study, the observed effects of the combination of these antibiotics, which have different antibacterial spectra and different sites of action on cell wall synthesis suggest synergistic action. This is supported by two different sets of data. First, the combination of cefsulodin and mecillinam produced an expansion of the antibacterial spectrum. This suggests that the combination of the two antibiotics does have a synergistic effect against several bacterial species. Second, the effect of the combination of the two antibiotics when examined by the checkerboard dilution method against selected bacterial strains, the fixed combination method against many clinical isolates, and the killing kinetics against certain bacterial strains also suggests a synergistic effect. In particular, a marked synergistic effect was observed on $S$. marcescens and $P$. mirabilis. The results in the fixed combination test were interesting, because the combination rates used in the test were established by the dose of each antibiotic used clinically and was not an optimal ratio resulting from the checkerboard dilution test. The killing kinetic test is the most prominent method for the analysis of the effect of combination but is an unsuitable method for studying effects against many strains.

The effect of a combination of mecillinam and broad spectrum $\beta$-lactam antibiotics has been reported on many members of Enterobacteriaceae and Bacteroides $\mathrm{sp}^{19 \sim 27)}$. However, this effect is not universal and the mode of action involved is not clear. The antibacterial activity of $\beta$-lactam antibiotics against Gram-negative bacteria is thought to be the outcome of complex series of steps involving the permeability of the drug through the bacterial outer membrane, the stability to hydrolysis by $\beta$-lactamases and the inhibitory activity of the drug against the peptidoglycan synthesizing enzymes ${ }^{28} 33$. The mechanism of action of antibiotic combination has been classified into three types involving increased entry of the antibiotics into the organism in order to reach their sites of activity, preventing of the inactivation of one antibiotic by the other, and action by the antibiotics at two different points in a biochemical pathway ${ }^{23)}$. Cefsulodin and mecillinam not only have a high resistance to hydrolysis by $\beta$-lactamases but a low affinity for enzymes ${ }^{34,35)}$. These findings suggest that the interaction between the two antibiotics and $\beta$ lactamases is not involved in the promotion mechanism. The two antibiotics have different sites of activity in the cell wall synthesizing mechanisms. It is possible that two antibiotics affect two different points in the pathway of cell wall synthesis. These possibilities will be investigated. 
A synergistic effect of cefsulodin plus mecillinam in vitro was observed on many bacterial species but in the in vivo test, a synergistic effect was observed on only two species, $S$. marcescens and $K$. pneumoniae. In mice infected with $S$. marcescens TN 66, the optimum ratio of the combination of the two antibiotics was different from that of the in vitro test. These results suggest that the effect of the combination must not only be tested in vitro but confirmed in vivo. SCHELD et al. ${ }^{\left.3{ }^{3}\right)}$ reported that the ampicillin-mecillinam combination was synergistic in a rabbit model of meningitis caused by $E$. coli but not synergistic in $K$. pneumoniae meningitis. More useful information may be presented by the test in model infections resembling clinical pictures. GRUNBerg and CleELAND ${ }^{5)}$ reported that there were suitable antibiotic partners for combination with mecillinam. The findings presented here suggest that for development of a synergistic effect by two antibiotics, a suitable antibiotic combination is an important factor and a synergistic effect may be found against certain bacterial species but never against all bacterial strains.

\section{Acknowledgments}

We gratefully acknowledge the helpful discussions of this manuscript by Prof. Dr. SHOICHIRo SuzUKI (Gifu University).

\section{References}

1) Tsuchiya, K.; M. Kondo \& H. Nagatomo: SCE-129, antipseudomonal cephalosporin: In vitro and in vivo antibacterial activities. Antimicr. Agents \& Chemoth. 13: 137 145, 1978

2) TsuchiYA, K. \& M. Kondo: Comparative in vitro activities of SCE-129, sulbenicillin, gentamicin and dibekacin against Pseudomonas. Antimicr. Agents \& Chemoth. 13: 536 539, 1978

3) Kondo, M. \& K. Tsuchiya: Comparative in vivo activities of cefsulodin, sulbenicillin, and gentamicin against Pseudomonas aeruginosa. Antimicr. Agents \& Chemoth. 14: 151 153, 1978

4) Tybring, L.: Mecillinam (FL 1060), a 6 3 -amidinopenicillanic acid derivative: In vitro evaluation. Antimicr. Agents \& Chemoth. 8: 266 270, 1975

5) Grunberg, E. \& R. Cleeland: In vivo activity of the $6 \beta$-amidinopenicillanic acid derivative, mecillinam, chemically linked or combined in varying ratios with 6-aminopenicillanic acid derivatives. J. Antimicrob. Chemoth. 3 (Suppl. B): 59 68, 1977

6) NAKAO, M.; T. YAMAZAKI \& K. TSUCHIYA: In vitro antibacterial activity of mecillinam and pivmecillinam. Chemotherapy (Tokyo) 25: 61 86, 1977

7) YAMAZAKI, T.; R. HAYASHI \& K. TsUCHIYA: In vivo antibacterial activity of pivmecillinam. Chemotherapy (Tokyo) 25: 87 93, 1977

8) Noguchi, H.; M. Matsuhashi \& S. Mitsuhashi: Comparative studies of penicillin-binding proteins in Pseudomonas aeruginosa and Escherichia coli. Eur. J. Biochem. 100: 41 49, 1979

9) Spratt, B. G.: Distinct penicillin binding proteins involved in the division, elongation and shape of Escherichia coli K 12. Proc. Nat. Acad. Sci., U.S.A. 72: 2999 3003, 1975

10) Mashimo, K.: Clinical experience with cefsulodin (SCE-129) in Pseudomonas aeruginosa infections in Japan. Program Abstr. No. 159, 18th Intersci. Conf. Antimicr. Agents Chemoth., Atlanta, Georgia, 1978

11) Wise, R.; M. Pippard \& D. S. Reeves: A laboratory and clinical investigation of mecillinam and its ester pivmecillinam in the treatment of urinary tract infections. J. Antimicrob. Chemoth. 3 (Suppl. B): 113 120, 1977

12) IsHIGAmI, J.: Clinical evaluation of pivmecillinam in acute simple cystitis: a comparative study with amoxycillin by a randomized double-blind technique. J. Antimicrob. Chemoth. 3 (Suppl. B): 129 135, 1977

13) Sabath, L. D.; C. E. McCall, N. H. Seighigel \& M. Finland: Synergistic penicillin combinations for treatment of human urinary-tract infections. Antimicr. Agents \& Chemoth. 1966: 149 155, 1967

14) Bushis, S. R. M. \& G. H. Hitching: Trimetoprim, a sulphonamide potentiator. Brit. J. Pharmacol. Chemother. 33: 72 90, 1968

15) Tsuchiya, K.; M. Kida, M. Kondo, H. Ono, M. TaKeuchi \& T. Nishi： SCE-963, a new broad-spectrum cephalosporin: In vitro and in vivo antibacterial activities. Antimicr. Agents \& Chemoth. 14: 557 568, 1978

16) Litchfield, J. T. \& F. Wilcoxon: A simple method of evaluating dose effect experiments. J. Pharmacol. Exp. Ther. 96: $99 \sim 113,1949$ 
17) Elion, G. B.; S. Singer \& G. H. Hitchings: Antagonists of nucleic acid derivatives. J. Biol. Chem. 208: $477 \sim 488,1954$

18) Ein, M. E.; N. J. Smith, J. F. Aruffo, M. S. Heerema, M. W. Bradshaw \& T. W. Williams, Jr.: Susceptibility and synergy studies of methicillin-resistant Staphylococcus epidermidis. Antimicr. Agents \& Chemoth. 16: $655 \sim 659,1979$

19) Greenwood, D. \& F. O'Grady: The two sites of penicillin action in Escherichia coli. J. Infect. Dis. 128: $791 \sim 794,1973$

20) Tybring, L. \& N. H. Melchior: Mecillinam (FL 1060), a 6 $\beta$-amidinopenicillanic acid derivative: Bactericidal action and synergy in vitro. Antimicr. Agents \& Chemoth. 8: 271 276, 1975

21) Grunberg, E.; R. Cleeland, G. Beskid \& W. F. Delorenzo: In vivo synergy between 6 $\beta$-amidinopenicillanic acid derivative and other antibiotics. Antimicr. Agents \& Chemoth. 9: 589 594, 1976

22) Baltimore, R. S.; J. O. Klein, C. Wilcox \& M. Finland: Synergy of mecillinam (FL 1060) with penicillin and cephalosporins against Proteus and Klebsiella, with observations on combinations with other antibiotics and against other bacterial species. Antimicr. Agents \& Chemoth. 9: 701 705, 1976

23) New, H. C.: Synergy of mecillinam, a beta-amidinopenicillanic acid derivative, combined with betalactam antibiotics. Antimicr. Agents \& Chemoth. 10: 535 542, 1976

24) New, H. C.: Mecillinam-an amidino penicillin which acts synergistically with other $\beta$-lactam compounds. J. Antimicr. Chemother. 3 (Suppl. B): 43 52, 1977

25) Chattopadhyay, B. \& I. Hall: In vitro combination of mecillinam with cephradine or amoxycillin for organisms resistant to single agents. J. Antimicrob. Chemother. 5: 549 553, 1979

26) Trestman, I.; D. Kaye \& M. E. Levison: Activity of semisynthetic penicillins and synergism with mecillinam against Bacteroides species. Antimicr. Agents \& Chemoth. 16: 283 286, 1979

27) Perea, E. J.; J. C. Palomares \& M. C. Garcia-Iglesias: In vitro evaluation of new penicillins and cephalosporins upon Pseudomonas aeruginosa and their interaction with mecillinam. Chemotherapy 26: 282 288, 1980

28) PARK, J. T.; J. R. Edwards \& E. M. Wise, Jr.: In vivo studies on the uptake and binding of $\beta$-lactam antibiotics in relation to inhibition of cell wall synthesis and cell death. Ann. N. Y. Acad. Sci. 235: 300 309, 1974

29) Costerton, J. M. \& E. J. Chang: The role of the bacterial cell envelope in antibiotic resistance. J. Antimicrob. Chemoth. $1: 363 \sim 377,1975$

30) Davies, J.: General mechanisms of antimicrobial resistance. Rev. Infect. Dis. 1: 23 27, 1979

31) Richmond, M. H.: $\beta$-Lactam antibiotics and $\beta$-lactamase: Two sides of a continuing story. Rev. Infect. Dis. 1: $30 \sim 36,1979$

32) Trpper, D. J.: Mode of action of $\beta$-lactam antibiotics. Rev. Infect. Dis. 1: 39 53, 1979

33) Tomaz, A.: From penicillin-binding proteins to the lysis and death of bacteria: A 1979 view. Rev. Infect. Dis. $1: 434 \sim 464,1979$

34) Okonogi, K.; M. Kida, M. Yoneda, J. Itoh \& S. Mitsuhashi: SCE-129, a new antipseudomonal cephalosporin and its biochemical properties. pp. 838 841. Ed., W. SiegenthaleR \& R. Lüthy, Current Chemotherapy Amer. Soc. Microbiol., American Society for Microbiology, Washington, D.C., 1978

35) Richmond, M. M.: In vitro studies with mecillinam on Escherichia coli and Pseudomonas aeruginosa. J. Antimicrob. Chemoth. 3 (Sppl. B): 29 39, 1977

36) Scheld, W. M.; F. N. Fink, P. D. Feltcher \& M. A. Sande: Mecillinam-ampicillin synergism in experimental Enterobacteriaceae meningitis. Antimicr. Agents \& Chemoth. 16: $271 \sim 276,1979$ 